Lindegren, C. C. \& Lindegren, G. (1951). J. gen. Microbiol. 5, 885-893.

\title{
Tetraploid Saccharomyces
}

\author{
By C. C. LINDEGREN AND GERTRUDE LINDEGREN \\ Southern Illinois University, Carbondale, Illinois
}

\begin{abstract}
SUMMARY: A yeast hybrid heterozygous for many alleles produces an occasional ascus in which instead of the expected 2 dominant: 2 recessive segregations one finds 4 dominant: 0 recessive ratios for most of the characters. The single ascospore cultures are often incapable of copulating with either mating type. Genetical analysis has revealed that such asci contain ascospores which are diploid and heterozygous for the mating type alleles and other characters. The single ascospore cultures, heterozygous for mating type, may sporulate and by characterization of their progeny the genotype of the ascospore can be determined. Other diploid cultures originating from single ascospores are homozygous for mating type. Cells from these cultures (unlike the diploid cells heterozygous for mating type) are capable of mating, and when mated with similar cells of complementary mating type produced tetraploid yeasts. Triploids were produced by mating diploids with haploids. The tetraploid segregates to produce the expected tetrad types and gametic ratios. Some sporulating cultures produced unexpected types of asci for characters controlling sugar fermentation but interpretation of their significance is not feasible since our customary control of breeding procedure does not apply in the analysis of diploid cultures which sporulate on ordinary agar slants.
\end{abstract}

\section{METHODS}

Safeguards in the breeding procedure. We have developed a breeding procedure which excludes errors due to intervening sporulations and copulations between the generations which are analysed. In the earlier phases of this work single ascospore cultures which produced spores were not unusual (Lindegren \& Lindegren, 1944). A rigid programme of selection was established and sporulating haplophase cultures were never used as parent breeding stocks. Diplophase cultures were never maintained as sources for breeding stocks since sporulation would be expected to occur and would quickly render them heterogeneous. Non-sporulating single ascospore haploid cultures were preferred as breeding stocks, since a minimum of variation occurs when they are carried on a rich nutrient medium by heavy transfers. A mating is made by mixing two heavy inocula of these cultures in a small amount of broth and transferring the cells to presporulation agar as soon as zygotes appear. On the presporulation agar, growth of both haplophase and diplophase occurs but spores are not produced. Most of the cells from the presporulation agar are transferred to gypsum. The immediate descendants of the zygotes sporulate while the haplophases do not. Each haploid culture used in a mating is carefully tested for its ability to sporulate by growth on presporulation agar followed by transfer to gypsum. Those which show any tendency to sporulate under these conditions are not used.

The advantages of our procedure are as follows: (1) Non-sporulating strains (haplophases) constitute the breeding stocks. (2) The composition of the 
presporulation medium permits the zygotes to multiply in the absence of sporulation. (3) The asci are formed on gypsum where germination of the spores does not occur and, since they are picked directly from the gypsum and dissected, intervening copulations are excluded. (4) The control of the time factor in this process may be as important an advantage as any other. Copulations begin about $6 \mathrm{hr}$. after the mixtures are made and the hybrids are complete after $12 \mathrm{hr}$. After $24 \mathrm{hr}$. on presporulation agar, which has been designed especially to hasten sporulation, the cells are transferred to gypsum and sporulate within $12 \mathrm{hr}$. Many current procedures require 10 days or 2 weeks to produce spores, and the disadvantage of such an extended period lies in the fact that critical observation cannot be maintained and therefore control of the procedure is lost.

It should be pointed out that sporulation of the diploid culture followed by copulation between spores or gametes could quickly lead to a new genotype. In such a case if only a few markers are used, the ratio of asci containing four dominants to those containing a fewer number might be nothing more than an evidence of the frequency with which intervening copulations had occurred. Assurance against this type of accident may be obtained by employing genetical stocks with large numbers of markers. Our current breeding stock (Lindegren \& Lindegren, 1951) is heterozygous for genes marking seven loci controlling sugar fermentation, six loci controlling B vitamin synthesis, eight loci controlling amino-acid synthesis, two loci controlling adenine synthesis and two controlling uracil synthesis.

Tetrad analysis is the procedure which gives the fungi a special advantage in genetical study. In the genetics of higher organisms, the events occurring during the reduction division must be inferred on the basis of the distribution of the various genetical characteristics among the progeny of a mating. For example, segregation of chromosomes in the sperm mother cell of a diploid male is inferred from the characteristics displayed by his offspring after mating to one or many females. In yeast, such inference is unnecessary since the four spores which are the homologues of the four sperm can be separated and grown to maturity as individual clones. Furthermore, the recovery among the four spores from a single ascus of characters originating from both parents is unequivocal proof of the hybrid nature of the diploid cell from which the ascus was produced.

\section{RESULTS}

The present paper analyses a type of deviation from the expected 2:2 Mendelian segregation in Saccharomyces asci due to the spontaneous diploidization of the ascospores. This phenomenon has also been described by Roman, Hawthorne \& Douglas (1951). In addition, it reports the controlled production of triploid and tetraploid hybrids by the use of diploid gametes originating from diploid ascospores homozygous for mating type.

Spontaneous diploidization of ascospores. The analysis of hybrid $10868 \times 11056$ (Table 1) resulted in the discovery of an exceptional ascus in which most of the segregants showed the dominant phenotype. 10868 was a white and 11056 was a pink (adenine-dependent) culture. Two of the twenty-seven asci were 
Table 1. Analysis of a hybrid heterozygous for ten loci in which one ascus containing spontaneously diploid ascospores occurred

Culture

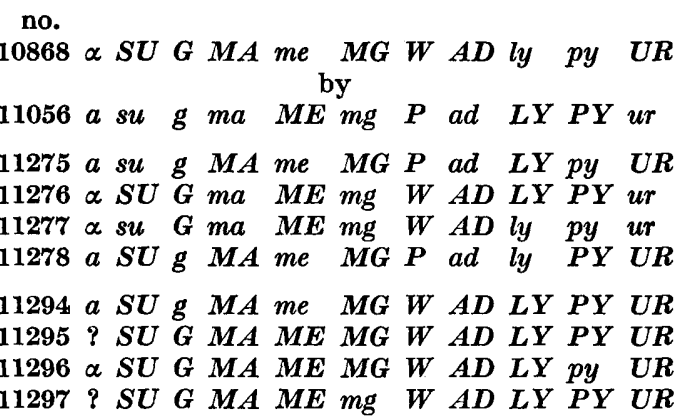

The mating type genes in yeast have been designated respectively $a$ and $\alpha$. Cultures of mating type $a$ are able to copulate with $\alpha$, but $a$ cultures are not able to copulate with $a$, nor is $\alpha$ able to copulate with $\alpha$. Each regularly segregating ascus normally contains two $a$ and two $\alpha$ spores. The diploid cultures $(a / \alpha)$ produced by copulations between $a$ and $\alpha$ cells are incapable of copulating with either mating type and cannot be classified with regard to mating type. Further analysis revealed that 11295 and 11297 were $a / \alpha ;(?)$ indicates their inability to copulate with a known tester.

The letters $S U, G, M A, M E, M G$ indicate the ability of the cultures to produce gas in Durham tubes of nutrient medium which contain respectively: sucrose, galactose, maltose, melibiose or $\alpha$-methylglucoside as the sole carbohydrate sources. The corresponding small letters indicate their inability to produce measurable amounts of gas under these circumstances. The letters $A D, L Y, U R, P Y$, indicate the ability of the cells to grow in Burkholder's medium (Burkholder \& Moyer, 1943) lacking the following nutrilites, respectively: adenine, lysine, uracil and pyridoxin. The small letters indicate inability to grow under these circumstances. The ad cultures are pink $(P)$.

exceptional. One ascus produced three pink and a single white culture, while the other (11294-11297) produced four white cultures, all of which were adenine-independent. Ascus 11275-11278 is one of a sample of five asci which were characterızed for all ten loci and which showed regular segregation. The other asci were merely characterized for pink $(P)$ and white $(W)$ with the results shown below:

$$
\begin{array}{llllr}
\boldsymbol{P} & \boldsymbol{P} & W & W & \mathbf{1 7} \\
\boldsymbol{P} & \boldsymbol{P} & W & - & \mathbf{2} \\
\boldsymbol{P} & - & W & W & \mathbf{5} \\
\boldsymbol{P} & W^{*} & W & W & 1 \\
\boldsymbol{P} & \boldsymbol{P} & \boldsymbol{P} & W & 1 \\
W & W & W & W & 1
\end{array}
$$

Among the asci in which only three spores survived no exceptions were encountered, although it is obvious that this does not establish them as standard asci. Corisiderable variation from light pink to obvious pink was observed so that it is possible, especially in the case of the white culture marked with an asterisk, that the colour was too light to be clear, or that an overgrowth of white cells may have obscured the pink phenotype. The reverse of this situation has not occurred. Under our conditions a genetically white culture does not take on a pink appearance. 
Work in this laboratory has consistently confirmed the relation between pink colour and adenine or hypoxanthine dependence. Pink cultures have always proved to be adenine-dependent, and occasional cultures which were pink or apparently white, may also be adenine-dependent, but no adenineindependent culture is ever pink. In yeast (as in Neurospora) adenine-dependent $(a d)$ cultures are pink owing to the accumulation and apparent polymerization of a precursor of adenine. Ability to complete the synthesis of adenine results in the removal of the precursor and the absence of the pink colour. Hybrids heterozygous for $A D / a d$ are white. The fact that one parent was pink and the other white suggested that the four spores of ascus 11294-11297 were diploid and heterozygous for $A D / a d$. The appearance of pyridoxin-independent, lysineindependent and melibiose fermenters in this ascus clearly demonstrates that it is not an illegitimate offspring of 10868.

Table 2. Phenotypes of the ascospores derived from 4-spored asci of culture $11295 S U G M A M G W A D$ (only three spores survived in the asci)

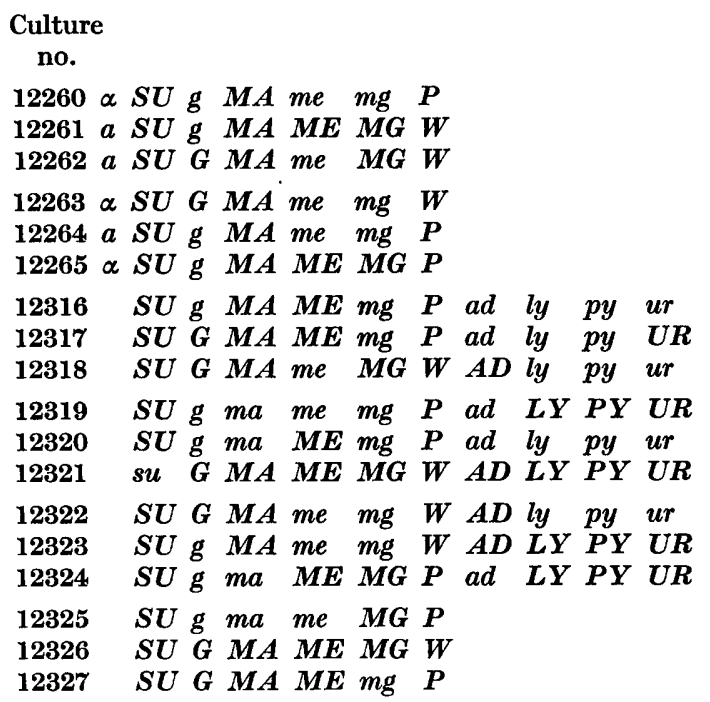

For explanation of symbols see Table 1 .

Segregation of a diploid culture obtained from a single ascospore. Culture 11295 sporulated on the agar slant without recourse to either presporulation agar or gypsum. Six 4-spored asci (only three spores germinated from each) were analysed (Table 2). It should be emphasized that under these conditions our rigid control of the genetical method does not apply. If copulations occur

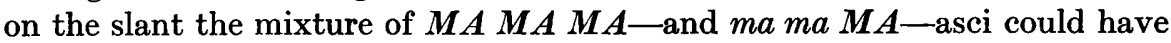
alternative interpretations; if the diploid cells were $M A / m a$ an intervening sporulation could have produced a copulation between two $M A$ segregants producing the $M A M A M A M A$ asci. The appearance, however, of any recessive alleles is evidence supporting the view that the original culture was 
heterozygous. On this basis 11295 was heterozygous for all ten loci with the possible exception of $S U / s u$.

Table 3. Phenotype of ascospores derived from 4-spored asci of culture

11297. SU GMA ME mg (in two asci only three spores survived)

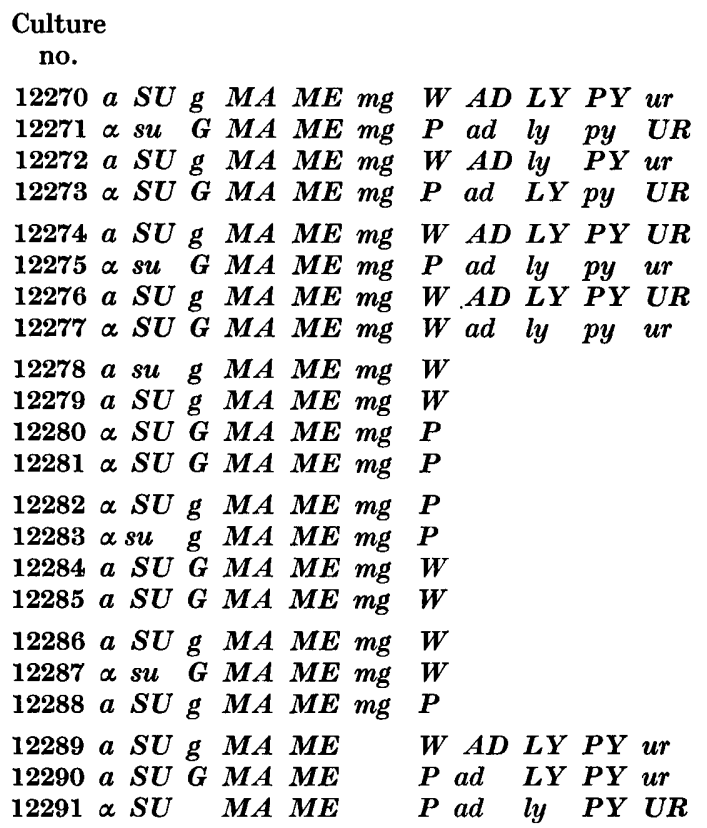

For explanation of symbols see Table 1.

Culture 11297 was also analysed (Table 3); and the same arguments apply. Six 4-spored asci (in two only three spores germinated) were cultured. Irregularities were encountered in the $\mathrm{SU} / s u$ segregation, but this phenomenon requires further study, since: (1) the dominant $S U$ parent arose from a pedigree in which irregular segregation of the ability to ferment sucrose occurred; (2) Hestrin \& Lindegren (1950) have shown that sucrase may be a concealed enzyme which becomes overt on special occasions, and Gilliland (1949) has shown that at least three different alleles may control sucrose fermentation.

Culture 11297 appears to be heterozygous for $a / \alpha, S U / s u, G / g, A D / a d, L Y / l y$, $P Y / p y$ and $U R / u r$, and homozygous for $M A, M E$ and $m g$.

These facts are consistent with the view that the ascospores in ascus 1129411297 are diploid. Cultures 11294 and 11296 do not sporulate but the genotypes, shown below, may be inferred from the data obtained from 11295 and 11297.

$\begin{array}{lllllllllll}11294 & a / a & S U / s u & g / g & M A / ? & m e / m e & M G / ? & A D / a d & L Y / l y & P Y / P Y & U R / u r \\ 11295 & a / \alpha & S U / s u & G / g & M A / m a & M E / m e & M G / m g & A D / a d & L Y / l y & P Y / p y & U R / u r \\ 11296 & \alpha / \alpha & S U / s u & G / G & M A / ? & M E / m e & M G / ? & A D / a d & L Y / l y & p y / p y & U R / u r \\ 11297 & a / \alpha & S U / s u & G / g & M A / M A & M E / M E & m g / m g & A D / a d & L Y / l y & P Y / p y & U R / u r\end{array}$


When all four diploid spores are of the dominant phenotype, as is the case with $S U, M A, A D, L Y, U R$ the cultures may all be heterozygous. Culture 11294 is apparently $g / g$; cultures 11295 and 11297 are $G / g$, suggesting that 11296 is $\boldsymbol{G} / \boldsymbol{G}$.

Since all four members of the tetrad are diploid the change must have occurred preceding meiosis.

\section{Production of a triploid and tetraploid zygotes}

Culture 11294 has been inferred to be $a / a$ and 11296 to be $\alpha / \alpha$; these cultures are therefore diploid gametes of contrasting mating type. They were mated with each other and with haploid gametic clones. When mated to haploids, triploid cells were produced and when mated with each other they produced tetraploid cells (Fig. 1). The cells of triploid and tetraploid cultures are large compared with those of standard diploid and haploid cultures and with diploid gametes.

Large yeast cells have been obtained by other workers following treatment with a variety of agents, and it has been suggested that the large cells arose through polyploidy (Bauch, 1942; Thaysen \& Morris, 1943). Duraiswami \& Subramaniam (1950) have argued, however, that cell-size is not an adequate criterion for the identification of polyploid series in yeast. They have proposed that fluctuations can be identified by the appearance of the colonies and by variations in the number of chromatic particles found within the cell. Duraiswami \& Subramaniam's cytological data concerning chromatic particles do not support the view that changes in ploidy occur, since they did not locate the particles with regard to either the centrosome, the nuclear vacuole, or the cytoplasm. The locations of the Feulgen-positive structures in the yeast cell are discussed in detail by Lindegren \& Rafalko (1950). The controlled production of triploid and tetraploid yeasts should not be taken as supporting Subramaniam's views on variations of ploidy; the inadequacy of his views has been exposed by Winge (1951) in a recent critique.

\section{Tetrad analysis of the tetraploid}

The tetraploid hybrid between 11294 and 11296 was analysed (Table 4). The general excess of dominant characteristics among the segregants from the tetraploid is expected. The hybrid is $A D A D a d a d$. The $a d$ cultures are pink $(P)$ and the $A D$ cultures are white $(W)$. Segregations for $P$ and $W$ are not in contradiction of expectation. The zygote is presumed to be $M E$ me me me. On this basis the third ascus is not explicable unless an intervening sporulation may have occurred on the agar slant, a possibility which cannot be excluded in dealing with cultures which sporulate on the agar slant. 


\section{Tetrad analysis of diploid segregants from the tetraploid}

The cultures of the first three asci were tested for their ability to sporulate with the results indicated in Table 4. Single ascospore cultures from 12369 and 12370, both of which were me, yielded only me offspring (Table 5). Culture 12380 was a fermenter of galactose. An excess of $g$ ascospores occurred

Table 4. Segregation of the tetraploid $(11296 \times 11294)$

\begin{tabular}{|c|c|c|c|}
\hline $\begin{array}{c}\text { Culture } \\
\text { no. }\end{array}$ & & & Spores \\
\hline 12369 & $? S U G M A m e$ & $M G W$ & + \\
\hline 12370 & $? S U G M A m e$ & $M G W$ & + \\
\hline 12371 & ? $S U G M A M E$ & $M G W$ & + \\
\hline 12372 & ? $S U G M A m e$ & $M G P$ & - \\
\hline 12373 & $\propto S U G M A M E$ & $M G W$ & - \\
\hline 12374 & $\alpha s u \quad g \quad M A M E$ & $M G P$ & - \\
\hline 12375 & $a s u \quad G M A m e$ & $M G W$ & - \\
\hline 12376 & $a S U g \quad M A m e$ & $M G W$ & - \\
\hline 12377 & ? $S U G M A M E$ & $M G P$ & - \\
\hline 12378 & $? S U G M A m e$ & $M G W$ & - \\
\hline 12379 & ? SU G MA ME & $\boldsymbol{M G} \boldsymbol{W}$ & + \\
\hline 12380 & ? $S U G M A M E$ & $M G W$ & + \\
\hline 12381 & $S U G M A M E$ & $M G W$ & \\
\hline 12382 & $S U G M A m e$ & $M G W$ & \\
\hline 12383 & $S U G M A m e$ & $M G W$ & \\
\hline 12384 & $S U g M A M E$ & $M G P$ & $a d$ \\
\hline 12385 & $S U G M A m e$ & $M G W$ & $A D$ \\
\hline 12386 & $S U G M A M E$ & $M G W$ & $A D$ \\
\hline 12387 & $S U g M A M E$ & $M G P$ & $a d$ \\
\hline 12388 & $S U G M A m e$ & $\boldsymbol{M G} \boldsymbol{W}$ & $A D$ \\
\hline 12389 & $S U G M A M E$ & $M G W$ & $A D$ \\
\hline 12394 & $S U G M A m e$ & $M G$ & \\
\hline 12395 & $S U G M A M E$ & $M G$ & \\
\hline 12396 & $S U G M A M E$ & $M G$ & \\
\hline
\end{tabular}

For explanation of symbols see Table 1 .

in four asci. The third ascus from 12380 was me me me me. The parent culture was a melibiose fermenter. Culture 12380 which arose as a segregant from the tetraploid zygote exhibited the expected segregation of $P$ and $W$, but the segregations of fermentative ability toward galactose and melibiose were exceptional. Exceptions in this case may, or may not, indicate exceptions to Mendelian rules, since, as has been pointed out, our customary control over intervening life cycles is not feasible when the diploid cultures sporulate on agar slants.

This work was supported by grants from Southern Illinois University, AnheuserBusch, Inc., and the United States Public Health Service. 
Table 5. Tetrad analyses of three diploid ascospore cultures derived from the tetraploid

Culture 12369: $S G M A$ me $M G W$ spores

Culture

no.

12404 a SU G MA me MG

$12405 \propto S U \quad M A m e \quad M G$

12406 a SU G $M A$ me $M G$

Culture 12370: $S$ G $M A$ me $M G W$ spores

$\begin{array}{lllllllll}12390 & \propto & S U & G & M A & m e & M G & P & a d \\ 12391 & a & s u & g & M A & m e & M G & P & a d \\ 12392 & \propto & s u & G & M A & m e & m g & W & A D \\ 12393 & a & S U & g & M A & m e & m g & W & A D \\ 12401 & S U & G & m a & m e & M G & \\ 12402 & S U & G & M A & m e & m g \\ 12403 & S U & g & M A & m e & M G \\ 12407 & s u & G & m a & m e & m g \\ 12408 & s u & g & m a & m e & m g \\ 12409 & S U & g & M A & m e & M G \\ 12410 & S U & G & M A & m e & M G\end{array}$

Culture 12380: $S G M A M E M G W$ spores

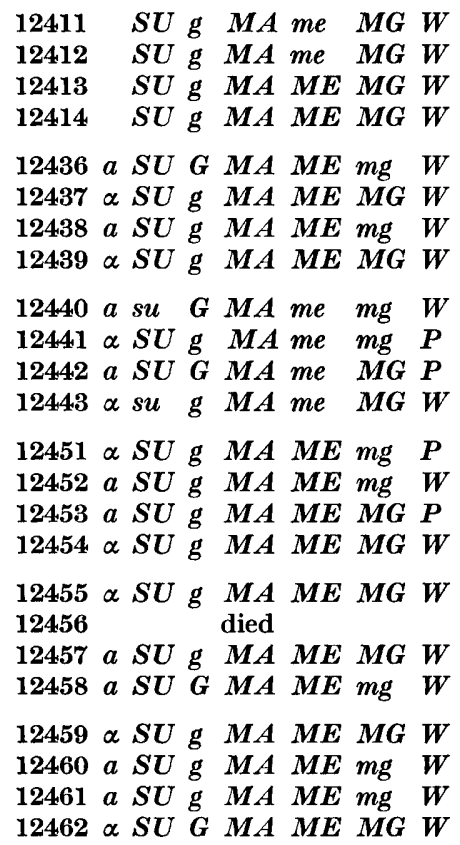

For explanation of symbols see Table 1 . 

Journal of General Microbiology, Vol. 5, No. 5

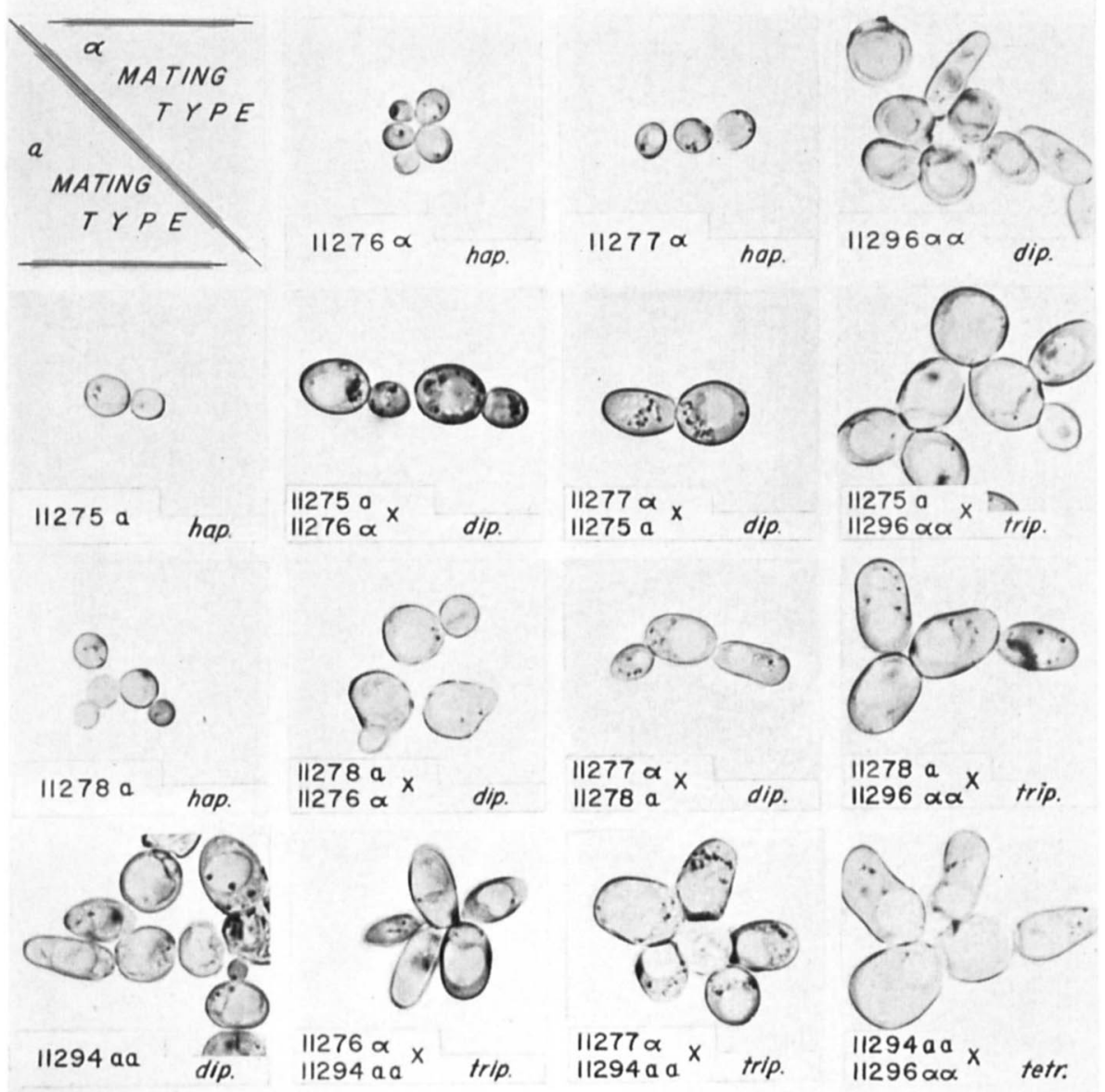

C. C. Lindegren \& Gertrude Lindegren-Tetraploid Saccharomyces. Plate 1 


\section{REFERENCES}

BAUCH, R. (1942). Über Beziehungen zwischen polyploidisierenden, carcinogenen und phytohormonalen Substanzen. Naturwissenchaften, 30, 420-1.

Burkholder, P. R. \& Moyer, D. (1943). Vitamin deficiencies of fifty yeasts and molds. Bull. Torrey bot. $\mathrm{Cl}$. 70, 372-7.

Duraiswami, S. \& Subramaniam, M. K. (1950). Reversal of some chromosomal mutations in yeasts. Cellule, $53,215-56$.

Gillilland, R. B. (1949). A yeast hybrid heterozygotic in four fermentation characters. C.R. Lab. Carlsberg, 24, 347-56.

Hestrin, Shlomo \& Lindegren, C. C. (1950). Carbohydrases in Saccharomyces haploid stocks of defined genotype. I. Fermentation and hydrolysis of alphaglucosides by yeast 6233. Arch. Biochem. 29, 315-33.

Lindegren, C. C. \& Lindegren, G. (1944). Instability of the mating type alleles in Saccharomyces. Ann. Mo. bot. Gdn, 31, 203-16.

Lindegren, C. C. \& Rafalko, M. M. (1950). The structure of the nucleus of Saccharomyces Bayanus. Exp. Cell Res. 1, 169-87.

Lindegren, C. C. \& Lindegren, G. (1951). Linkage relations in Saccharomyces of genes controlling the fermentation of carbohydrates and the synthesis of vitamins, amino-acids, and nucleic acid compounds. Indian Phytopathology (in the Press).

Roman, H., Hawthorne, D. C. \& Douglas, H. C. (1951). Polyploidy in yeast and its bearing on the occurrence of irregular genetic ratios. Proc. Nat. Acad. Sci., Wash., 37, 79-84.

Thaysen, A. C. \& Morris, M. (1943). Preparation of a giant strain of Torulopsis utilis. Nature, Lond., 152, 526-8.

Winge, 0 . (1951). The relation between yeast cytology and genetics: a critique. C.R. Lab. Carlsberg. 25, 85-99.

\section{EXPLANATION OF PLATE}

Plate 1. A 'checkerboard' diagram with the gametes forming two outer rows. The $a$ gametes form the column at the left and the $\alpha$ gametes form the top row. Each hybrid is located at the intersection of a row and a column. The tetraploid hybrid between the $a a$ and the $\alpha \alpha$ gametes is found in the lower right-hand corner. Four diploid hybrids, four triploid hybrids, and a single tetraploid hybrid were produced by matings between the six different gametes; four of the gametes are haploid and two are diploid. The haploid gametes are the smallest cells; the diploid gametes are considerably larger and are approximately the same size as the diploid hybrids. Matings between the haploid and diploid gametes produced four different triploid hybrids which are much larger than the diploid cultures. The single tetraploid produced by mating two diploid gametes is the largest hybrid. Photographs by Frank L. Kennedy, jun. Magnification $\times 3060$.

(Received 28 March 1951) 